PROCEEDINGS OF THE

AMERICAN MATHEMATICAL SOCIETY

Volume 132, Number 1, Pages 33-35

S 0002-9939(03)07218-6

Article electronically published on July 17, 2003

\title{
MONOTONE MATRIX FUNCTIONS OF SUCCESSIVE ORDERS
}

\author{
SUHAS NAYAK
}

(Communicated by Jonathan M. Borwein)

\begin{abstract}
This paper extends a result obtained by Wigner and von Neumann. We prove that a non-constant real-valued function, $f(x)$, in $C^{3}(I)$ where $I$ is an interval of the real line, is a monotone matrix function of order $n+1$ on $I$ if and only if a related, modified function $g_{x_{0}}(x)$ is a monotone matrix function of order $n$ for every value of $x_{0}$ in $I$, assuming that $f^{\prime}$ is strictly positive on $I$.
\end{abstract}

A real-valued function, $f$, is matrix monotone of order $n$ over an interval, $I$, if, for two symmetric $n \times n$ matrices, $A, B$, with eigenvalues in $I, A \leq B$ implies that $f(A) \leq f(B)$. Call the class of all such functions, $P_{n}(I)$. Many results on monotone matrix functions may be found in the monograph by Donoghue 2]. Of particular interest is the characterization of functions that are operator monotone, i.e., monotone of all orders $n$. We denote this class of functions by $P_{\infty}(I)$. Löwner 4 showed that functions that are operator monotone on $I$ are exactly those functions that are analytic with positive imaginary part in the upper half-plane (Pick functions) and that have an analytic continuation to the lower half-plane across $I$, where the continuation is by reflection. This is the content of Löwner's Theorem. Many different proofs of this theorem are known (see, e.g., Donoghue [2] for a survey of a number of them). Perhaps the least well-known proof of Löwner's Theorem is due to Wigner and von Neumann [6]. We prove an extension of one of the results in their work.

Define a modified function, $g_{x_{0}}(x)$ as follows:

$$
g_{x_{0}}(x)=-\frac{1}{f(x)-f\left(x_{0}\right)}+\frac{1}{f^{\prime}\left(x_{0}\right)\left(x-x_{0}\right)} .
$$

We define $g_{x_{0}}\left(x_{0}\right)$ to be the limit as $x \rightarrow x_{0}$ of $g_{x_{0}}(x)$, and this limit exists if $f$ is $C^{2}$ there, by L'Hôpital's Rule and Taylor's Theorem.

Theorem 1. Let $f$ be in $C^{3}(a, b)$ with the additional property that $f^{\prime}(x)>0$ for all $x \in(a, b) . \quad f$ is a monotone matrix function of order $n+1(n \geq 1)$ on an interval, $(a, b)$, if and only if $g_{x_{0}}$ is a monotone matrix function of order $n$ on the same interval for all $x_{0} \in(a, b)$.

Received by the editors August 25, 2002.

2000 Mathematics Subject Classification. Primary 15A48; Secondary 15A24, 47A63.

Key words and phrases. Monotone matrix functions, Löwner's Theorem, Sylvester's Determinant Identity.

This work was conducted as part of a senior thesis at the California Institute of Technology.

(C)2003 American Mathematical Society 
The assumptions in Theorem 1 are not too restrictive. It is known (see [2]) that $f \in P_{n}(a, b)$ implies $f \in C^{(2 n-3)}(a, b)$. Moreover, if $f$ is non-constant and in $P_{2}(a, b)$, then $f^{\prime}(x)>0$ for all $x \in(a, b)$. Since $P_{k}(a, b) \subset P_{j}(a, b)$ for all $k>j, f^{\prime}$ is strictly positive if $f \in P_{n}(a, b)$ with $n \geq 2$.

It should also be noted that Wigner and von Neumann [6] proved one half of Theorem 1, namely that $f$ monotone of order $n+1$ in $(a, b)$ implies that $g_{x_{0}}$ is monotone of order $n$ in $(a, b)$ for any $x_{0}$ in $(a, b)$.

Proof of Theorem 1. The divided differences of a function, $f$, may be defined as follows:

$$
\left[x_{i}, x_{j}\right]_{f}=\frac{f\left(x_{i}\right)-f\left(x_{j}\right)}{x_{i}-x_{j}}, \quad\left[x_{i}, x_{i}\right]_{f}=f^{\prime}\left(x_{i}\right) .
$$

It is well known that $f \in P_{n+1}(a, b)$ if and only if for any set of $n+1$ distinct points $x_{0}, x_{1}, x_{2}, \ldots, x_{n}$ of the interval $(a, b)$, the Pick matrix formed with the divided differences of $f, K_{i j}^{(f)}=\left[x_{i}, x_{j}\right]_{f}=a_{i j}$, is a positive semidefinite matrix. We therefore consider the Pick matrices of $f$ and $g_{x_{0}}$. It is straightforward to verify that for $x_{i}$ and $x_{j}$ different from $x_{0}$,

$$
\left[x_{i}, x_{j}\right]_{g_{x_{0}}}=\frac{a_{00} a_{i j}-a_{i 0} a_{j 0}}{f^{\prime}\left(x_{0}\right)\left(f\left(x_{i}\right)-f\left(x_{0}\right)\right)\left(f\left(x_{j}\right)-f\left(x_{0}\right)\right)} .
$$

Also, by L'Hôpital's Rule and Taylor's Theorem, it can be shown that $g_{x_{0}}\left(x_{0}\right)$ and $g_{x_{0}}^{\prime}\left(x_{0}\right)$ exist if $f \in C^{3}(a, b)$.

We consider the determinants of the principal submatrices of $K^{\left(g_{x_{0}}\right)}$. Consider the determinant of any one such submatrix. The term-by-term expansion of the determinant is a linear combination of fractions with the same denominator. This denominator is a product of terms of the form $\left(f\left(x_{i}\right)-f\left(x_{0}\right)\right)^{2}$ (for each row $i$ of $K^{\left(g_{x_{0}}\right)}$ in the submatrix) and a positive power of $f^{\prime}\left(x_{0}\right)$. Therefore this common denominator is positive. Factoring out this common denominator leaves the determinant of the corresponding submatrix of $L^{\left(g_{x_{0}}\right)}$, defined by $L_{i j}^{\left(g_{x_{0}}\right)}=a_{00} a_{i j}-a_{i 0} a_{j 0}$, which is just the matrix of the numerators of each entry in $K^{\left(g_{x_{0}}\right)}$. Denote the entries of $L^{\left(g_{x_{0}}\right)}$ by $b_{i j}$. Since the determinant of each submatrix of $K^{\left(g_{x_{0}}\right)}$ has the same sign as the determinant of the corresponding submatrix of $L^{\left(g_{x_{0}}\right)}$, it suffices to consider $L^{\left(g_{x_{0}}\right)}$ in what follows.

Suppose $f$ is monotone of order $n+1$. Let us now consider a $k \times k$ principal submatrix of $L^{\left(g_{x_{0}}\right)}, L_{s u b}^{\left(g_{x_{0}}\right)}$, containing diagonal entries $b_{n_{i} n_{i}}$. Using Sylvester's Determinant Identity ([5], [1]), we find that $\operatorname{det} L_{\text {sub }}^{\left(g_{x_{0}}\right)}=a_{00}^{k-1} \operatorname{det} K_{s u b}^{(f)}$, where $K_{\text {sub }}^{(f)}$ is the $(k+1) \times(k+1)$ principal submatrix of $K^{(f)}$ having diagonal entries $a_{00}$ and $a_{n_{i} n_{i}}$. Since $f$ is monotone of order $n+1$, $\operatorname{det} L_{s u b}^{\left(g_{x_{0}}\right)}$ is nonnegative, and therefore any Pick matrix of $g_{x_{0}}$ formed by a subset of the points $\left(x_{1}, x_{2}, \ldots, x_{n}\right)$ (with $x_{i} \neq x_{0}$ ) is a positive semidefinite matrix. If we take a limit of the positive semidefinite Pick matrices as $x_{i} \rightarrow x_{0}$, we get a positive semidefinite matrix. This is the first half of the theorem, and was the result used by Wigner and von Neumann in their continued fraction proof of Löwner's Theorem.

The converse is easily proved also. Suppose $g_{x_{0}} \in M_{n}(a, b) \quad \forall x_{0} \in(a, b)$. Consider any principal submatrix of $K^{(f)}$. If the submatrix is $1 \times 1$, then it is one of the diagonal elements, which are nonnegative since we know that $f^{\prime}$ is positive on $(a, b)$. Now consider a $k \times k$ principal submatrix of $K^{(f)}$, which is the Pick matrix associated to $f$ of the distinct points $x_{n_{1}}, x_{n_{2}}, \ldots, x_{n_{k}}\left(n_{i} \in\{1,2, \ldots, n\}\right)$. 
By another application of Sylvester's Determinant Identity, the determinant of this submatrix has the same sign as the determinant of the $(k-1) \times(k-1)$ submatrix of the Pick matrix associated to $g_{x_{n_{1}}}$ of the points $x_{n_{2}}, x_{n_{3}}, \ldots, x_{n_{k}}$. But these latter determinants are nonnegative, which means that $f$ is monotone of order $n+1$.

The question of gaps between classes of monotone matrix functions has been addressed in Donoghue [2], and, more recently, in an article by Hansen et al. [3]. The corollary below, which concerns such gaps, follows from Theorem 1.

Corollary 2. If $n \geq 2$, then $P_{n}(a, b)=P_{n+1}(a, b)$ implies $P_{n}(a, b)=P_{\infty}(a, b)$.

Proof. Suppose, for some $n \geq 2, P_{n}(a, b)=P_{n+1}(a, b)$. Take $f \in P_{n+1}(a, b)$. Then, $g_{x_{0}} \in P_{n}(a, b)$ for all $x_{0} \in(a, b)$, and since $P_{n}=P_{n+1}$, we have that $g_{x_{0}} \in P_{n+1}(a, b)$ for all $x_{0} \in(a, b)$, and, therefore, by our theorem, $f \in P_{n+2}(a, b)$. The corollary then follows by induction.

\section{ACKNOWLEDGEMENTS}

The author wishes to thank B. Simon for uncovering the Wigner and von Neumann continued fraction proof and the paper on which this research is based, and for many helpful discussions.

\section{REFERENCES}

1. A. G. Akritas, E. K. Akritas, and G. Malaschonok, Various proofs of Sylvester's (determinant) identity, Math. Comput. Simulation, 42 (1996), 585-593. MR 98c:15021

2. W. F. Donoghue, Jr., Monotone matrix functions and analytic continuation, Springer-Verlag, New York, 1974. MR 58:6279

3. F. Hansen, G. Ji, and J. Tomiyama, Gaps between classes of matrix monotone functions, preprint.

4. K. Löwner, Über monotone Matrixfunktionen, Math. Z., 38 (1934), 177-216.

5. J. J. Sylvester, On the relation between the minor determinants of linearly equivalent quadratic functions, Philosophical Magazine 1 (Fourth Series) (1851), 294-305.

6. E. Wigner and J. von Neumann, Significance of Loewner's theorem in the quantum theory of collisions, Ann. of Math., 59 (1954), 418-433. MR 16:25d

Department of Mathematics, Caltech, Pasadena, California

Current address: Department of Mathematics, Stanford University, Stanford, California 943052125

E-mail address: snayak@stanford.edu 